\title{
Hemodynamic Impact of Intrathecal versus Epidural Analgesia with Sufentanil in the Early Phase of Labor: A Randomized Controlled Trial \\ Grazia Meneghetti ${ }^{1}$, Alba Ripoll Gallardo ${ }^{2 *}$, Claudio Ripa ${ }^{1}$, Valeria Viarengo ${ }^{1}$, Sara Fracon ${ }^{1}$, Daniela Ferrante ${ }^{3}$ and $^{-}$ Francesco Della Corte ${ }^{1}$ \\ ${ }^{1}$ Maggiore Hospital School of Medicine, Italy \\ ${ }^{2}$ CRIMEDIM- Research Center in Emergency and Disaster Medicine - Università del Piemonte Orientale, Italy \\ ${ }^{3} \mathrm{CPO}$ Piemonte and Unit of Medical Statistics and Epidemiology Department of Medical Science, University of Eastern Piedmont, Novara, Italy
}

Received: February 13, 2017; Accepted:April 5, 2017; Published: April 24, 2017

*Corresponding author: Alba Ripoll Gallardo, CRIMEDIM- Research Center in Emergency and Disaster Medicine - Università del Piemonte Orientale, Via Lanino 1, PC 28100 Novara, Italy, Tel: +49 17622840 127; E-mail: alba.ripoll@med.uniupo.it

\begin{abstract}
Background: Combined spinal epidural analgesia (CSEA) has become a widespread technique in obstetric analgesia. Sufentanil is currently widely used in combination with a low concentration of local anaesthetic to reduce both motor block and local anesthetic requirements. While some studies have reported on the hemodinamic changes after the intrathecal administration of sufentanil in the early phase of labor, no randomized controlled trials have been performed. We compared hemodynamic impact, quality of the analgesia, safety, side effects and impact on labor and delivery after the administration of two equipotent doses of sufentanil given by intrathecal versus epidural route.
\end{abstract}

Methods: Nulliparous parturients with full-term uncomplicated pregnancies, in established first stage of labor and requesting analgesia were enrolled into this randomised-controlled, single blind, parallel study. Patients were equally randomised to receive either epidural (EA) (sufentanil 1,4 mcg ml-1-15ml) or CSEA(sufentanil $2,5 \mathrm{mcg} / 4 \mathrm{ml}$ ). At the reappearance of pain, analgesia was continued in both groups with the same protocol of epidural boluses of levobupivacaine + sufentanil.

Results: Seventy parturients were enrolled in the study (35 for each group). There was a minimal and non statistically significant decrease from the baseline systolic arterial pressure $(127,9 \pm 14,6$ $\mathrm{mmHg}$ in EA and $124,6 \pm 28,3 \mathrm{mmHg}$ in CSEA) with no differences between the two groups $(125,9+15,54 \mathrm{mmHg}$ in $\mathrm{EA}$ and $122 \pm 14,62$ mmHg in CSEA). In both groups we observed a reduction of NRS mean values at 15 minutes $(p<0,05)$ that was lower in CSEA group $(3,2 \pm 2,4$ vs $5,1 \pm 2,2$ in EA group). Pruritus was more common in CSEA while the total time for dilatation, duration of the expulsive period, delivery type, rates of instrumental deliveryand foetal heart rate abnormalities were similar in both groups

Conclusions: This study was unable to demonstrate any difference in the hemodynamic effects of neuraxial analgesia with sufentanil in the early phase of labor when the intrathecal ED50 and the epidural ED50 were compared. Better and faster levels of analgesia may be achieved with CSEA.

Keywords: Sufentanil adverse effects- labor analgesia- obstetric analgesia

\section{Abbreviations}

EA: epidural analgesia combined spinal epidural analgesia; CSEA: combined spinal epidural analgesia; ED50: median effective dose; NRS: numeric rating scale; SAP: systolic arterial pressure; FHR: fetal heart rate; FHT: fetal heart tracing

\section{Introduction}

Analgesic techniques for pain control during labor and delivery include spinal, epidural (EA) and combined spinal epidural analgesia (CSEA). Spinal route is effective but insufficient to provide analgesia for the whole duration of labor. Epidural analgesia effectively relieves pain but has been associated with prolonged labour, increased use of oxytocin and of instrumental deliveries and maternal hypotension [1]. CSEA combines the advantages of both epidural and spinal techniques and has become a widespread technique in obstetric analgesia [1, 2]. The administration of perimedullary opioids in combination with lower concentration of local anesthetic has been gaining popularity to maintain some degree of motor function during the labor [1,3,4] and reduce local anesthetic requirements [5]. In this regard, Sufentanil has emerged as one of the most useful drugs for pain relief due to its potency (5-6 times that of fentanyl) [6], longer analgesic effect [7] and relatively safe profile. However, opioids have also been associated with dose-related side effects such as pruritus, respiratory depression and maternal hypotension [8]. Some studies have attributed the hemodynamic changes observed after the intrathecal administration of sufentanil to a weak local anesthetic effect of this drug $[9,10]$. However, little data on the hemodynamic effects of sufentanil when given as single analgesic agent in different administration routes has been published so far [11]. The aim of this study was to compare the hemodynamic impact in the early phase of labor of intrathecal versus epidural analgesia with sufentanil. 


\section{Methods \\ Design}

This prospective, equally randomized-controlled, single blind study was conducted in a tertiary level, academic hospital.

\section{Ethics approval}

Ethical approval for this study (Ethical Committee n. 170/09) was provided by the Ethical Committee of the Azienda Ospedaliera Universitaria "Maggiore della Carità" Novara, Italy on December 18, 2009 - Chairperson: Dr. Gianfranco Zulian. This clinical trial study was registered as EudraCT:2009-017665-31.

\section{Participants}

After obtaining written informed consent, we enrolled nulliparous parturients in established first stage of labor $(\leq 5 \mathrm{~cm}$ cervical dilatation) with regular painful uterine contractions (at least two in 10 minutes and lasting about 50-60 seconds) that required analgesia and according to inclusion and exclusion criteria (Table 1).

\begin{tabular}{|c|c|}
\hline Inclusion Criteria & Exclusion Criteria \\
\hline Age $>18$ yrs & Maternal physical status (ASA) $>2$ \\
\hline $\begin{array}{l}\text { Obstetrically uncomplicated, } \\
\text { vertex presentation, } \\
\text { singleton pregnancies, at } \\
\text { term }\end{array}$ & Twin pregnancy \\
\hline Nulliparous & $\begin{array}{l}\text { Congenital or acquired coagulation } \\
\text { disease } \\
(\text { plts }<70000 \text { cell } / \mathrm{ml} \text {; INR }>1.2 \text { ) }\end{array}$ \\
\hline $\begin{array}{l}\text { Active early labor }(\leq 5 \mathrm{~cm} \\
\text { cervical dilatation })\end{array}$ & $\begin{array}{c}\text { Therapy with anticoagulant or } \\
\text { antiaggregant drugs }\end{array}$ \\
\hline $\begin{array}{l}\text { Maternal request for labor } \\
\text { analgesia }\end{array}$ & Systemic or local infection \\
\hline $\begin{array}{l}\text { Written informed consent } \\
\text { to study }\end{array}$ & Hemodynamic instability \\
\hline & $\begin{array}{c}\text { Pre-analgesia FHR (Fetal Heart Rate) } \\
\text { abnormalities }\end{array}$ \\
\hline
\end{tabular}

\section{Endpoints and sample size}

The primary endpoint of this study was to compare the hemodynamic impact in the early phase of labor of the median effective dose (ED50) of intrathecal sufentanil administered by CSEA versus ED50 of sufentanil administered by epidural route. Secondary endpoints were the evaluation of the quality of the analgesia, safety, side effects and impact on labor and delivery The number of patients ( 35 for group) was attributed to a power level of 0,80 with a $\mathrm{P}<0,05$ statistically significant. This number was obtained considering as good outcome a decreasing blood pressure of less than $20 \%$ from baseline value after 15 minutes from the administration of sufentanil and a value of coefficient of variation equal to 0,5 .

\section{Intervention}

In EA group, sufentanil was initially injected alone into the epidural space $(1,4 \mathrm{mcg} \mathrm{ml}-1-15 \mathrm{ml})$ while in CSEA group the initial bolus of sufentanil was performed intrathecally $(2,5 \mathrm{mcg} / 4$ $\mathrm{ml}$ ). Both doses were decided according to the median effective dose (ED50) of sufentanil for intrathecal and epidural adminis tration as published by Herman et al [12] and Capogna et al [6], respectively. At the reappearance of pain (Numeric Rating Scale (NRS) >4), analgesia was continued in both groups with the same protocol of epidural boluses of levobupivacaine + sufentanil according to cervical dilatation and conforming to our Institutional Protocol (Table 2).

\begin{tabular}{|c|c|c|}
\hline \multicolumn{3}{|l|}{$\begin{array}{l}\text { Table 2: Protocols of treatment for Epidural Analgesia and Combined } \\
\text { Spinal-Epidural Analgesia groups }\end{array}$} \\
$\begin{array}{c}\text { Cervical } \\
\text { dilatation } \quad \text { (cm) }\end{array}$ & Bolus (drug and doses) & $\begin{array}{c}\text { Volume } \\
\text { (ml) }\end{array}$ \\
\hline$\leq 5$ & $\begin{array}{c}\text { First epidural bolus } \\
\text { Sufentanil } 1,4 \mathrm{mcg} / \mathrm{ml}\end{array}$ & $15 \mathrm{ml}$ \\
\hline $5-7$ & $\begin{array}{c}\text { Levo-bupivacaine } 0.1 \% \\
+ \text { sufentanil } 0.5 \mathrm{mcg} / \mathrm{ml}\end{array}$ & $10 \mathrm{ml}$ \\
\hline $8-10$ & $\begin{array}{c}\text { Levo-bupivacaine } 0.125 \% \\
\text { Levo-bupivacaine } 0.15 \%\end{array}$ & $10 \mathrm{ml}$ \\
\hline Expulsive period & & $10 \mathrm{ml}$ \\
\hline
\end{tabular}

Combined Spinal-epidural analgesia

\begin{tabular}{|c|c|c|}
\hline $\begin{array}{c}\text { Cervical } \\
\text { dilatation (cm) }\end{array}$ & Bolus (drug and doses) & Volume (ml) \\
\hline$\leq 5$ & $\begin{array}{c}\text { Intrathecal bolus } \\
\text { Sufentanil 2,5 mcg }\end{array}$ & $4 \mathrm{ml}$ \\
\hline $5-7$ & $\begin{array}{c}\text { Epidural Levo-bupivacaine } 0.1 \% \\
\text { +sufentanil } 0.5 \mathrm{mcg} / \mathrm{ml}\end{array}$ & $10 \mathrm{ml}$ \\
\hline $8-10$ & $\begin{array}{c}\text { Epidural Levo-bupivacaine } \\
0.125 \%\end{array}$ & $10 \mathrm{ml}$ \\
\hline Expulsive period & $\begin{array}{c}\text { Epidural Levo-bupivacaine } \\
0.15 \%\end{array}$ & $10 \mathrm{ml}$ \\
\hline
\end{tabular}

Cervical dilatation, level of engaging part and the progress of labour phase were evaluated by the same obstetrician before the administration of each bolus. All parturients received 10 $\mathrm{mL} / \mathrm{kg}$ lactated Ringer's solution before analgesia was started. For both groups, the epidural space was identified at the L2-3 or L3-4 intervertebral space using the loss of saline resistance technique. In CSEA group, the subarachnoid administration was performed with a 27-G Pencan needle in the same interspace by using a special needle kit (Espocan $(+$ Docking system + Perifix $(-$ Soft-Tip B/Braun, Thuoy 18GØ; catheter 20GØ). The catheter was thereafter advanced cephalad for 2,5-3 cm as for EA group. No dose-test was performed. Intrathecal placement of the catheter was excluded by negative aspiration of cerebrospinal fluid in both groups. Technical incidents (as dura mater perforation) resulted in exclusion of the subject from the study.

\section{Primary Outcome Measures}

Hemodynamic parameters (Systolic and diastolic non invasive arterial blood pressure and heart rate) were recorded at 15 (minimum onset of action described for intrathecal administration)[13], 30 minutes from the first bolus and thereafter every 30 minutes until delivery. Maternal hypotension was defined as a decrease in systolic arterial pressure (SAP) greater than $20 \%$ from the baseline or a SAP absolute value below 100 
mm Hg [14].

\section{Secondary outcome measures}

Pain score (using the NRS) were recorded at 15 and 30 minutes intervals after each bolus of analgesic. Uterine activity was monitored using external tocography in combination with clinical assessment by the attending obstetrician. Tocographic monitoring of uterine activity, indications for oxytocin administration and emergence of uterine hyperactivity were recorded by the attending obstetrician. Fetal wellness was monitored using external cardiotocography until childbirth; pathological or non reassuring fetal heart rate (FHR), as defined by the classification of the Royal College of Obstetricians and Gynaecologists guidelines 2001 [15], were reported. Fetal heart rate abnormalities were managed conservatively (left lateral decubitus, oxygen by face mask, IV fluids and cessation of IV-oxytocin). Cesarean delivery was performed according to national guidelines [16]. The fetal heart tracing (FHT) of the period comprised between 20 minutes prior and 1 hour after administration of analgesia was considered and reviewed at the end of the study by two obstetricians independently. Discrepancies were referred to a third obstetrician and the prevailing interpretation was recorded. We also recorded the total duration of labor (time period from initial to full dilatation), labor analgesia (time period from the first bolus of analgesia to full dilatation) and delivery (time between complete dilatation and childbirth). Delivery mode, perineal lesions, need for episiotomy, Apgar score and side effects were recorded as well as other possible complications. The level of sensory block (pinprick) and the presence of motor block (Bromage score) were recorded [17]. Finally, we measured the total dose of opioids and local anesthetics administered.

\section{Randomization}

Patients were equally randomized to receive either EA or CSEA. The randomization protocol was provided by the Statis- tic Laboratory of our Institution. SAS software procedure "procplan” (Release 8.2, by SAS/STAT®, Institute Inc, Cary, NC, USA) was used to perform all statistical analyses. The blocked randomized assignment of treatments was applied using block size of 6 or 10 .

\section{Blinding}

Patients were randomly assigned and blinded to the study arm. This was made possible due to the adoption of the same CSEA set in both groups.

\section{Statistical analysis}

ANOVA for repeated measurements was used to assess the differences in mean blood pressure values and NRS at baseline and at subsequent 15 and 30 minutes. This method of analysis was preferred due to the multiple responses taken in sequence on each experimental unit. The objective was to examine and compare response trends over time. We achieved this by comparing treatments averaged over time and comparing measurement time within a treatment. The unstructured (UN) covariance matrix was used as correlation pattern among responses on the same subject. The model was used in this analysis was $\mu \mathrm{ik}=\mu+\alpha \mathrm{i}+\Gamma \mathrm{k}+(\alpha \Gamma)$ ik where $\mu$ is the overall mean, $\alpha$ is the "group effect", $\Gamma$ is the "time effect" and ( $\alpha \Gamma)$ the "group x time effect" [18].

For not normally distributed data, we used logarithmic transformations. Non parametric Mann-Whitney test was used if the assumption of normality underlying test T-Student was violated. The chi-square test or Fisher's exact test were used to compare the proportions.

\section{Results}

Between March and December 2010, 165 parturients received neuroaxial analgesia for labor; 95 were not eligible to the study (50 did not sign the informed consent and 45 did not meet the inclusion criteria). Seventy parturients were enrolled in the study, 35 for each group; none was excluded from the analysis (Figure 1).

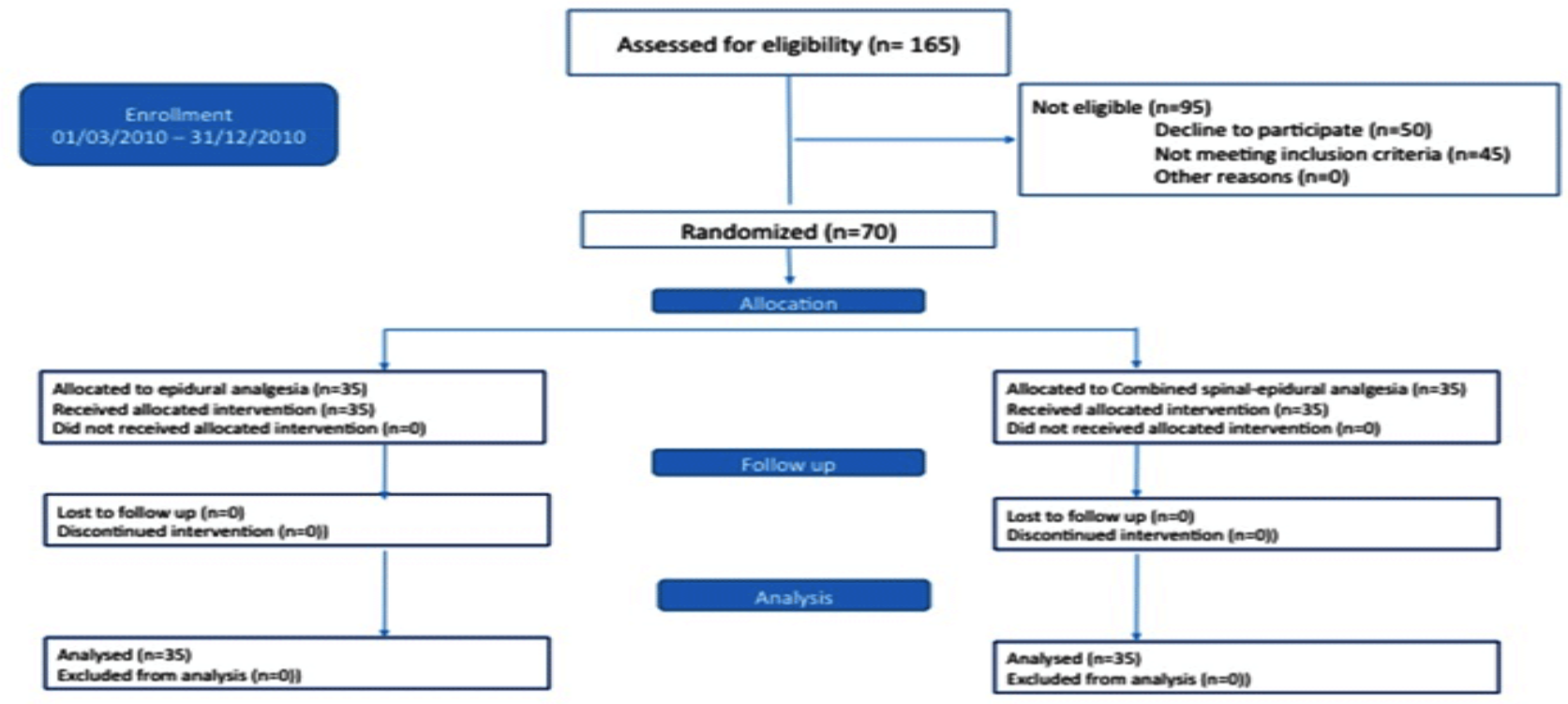

Figure 1: Consort Flow Chart

Citation:Alba Ripoll Gallardo, et.al. (2017) Hemodynamic Impact of Intrathecal versus Epidural Analgesia with Sufentanil in 


\section{Baseline characteristics}

No differences were found in demographic and clinical data. Labor was spontaneous in 21 parturients in EA group and 22 in CSEA group. Bishop index and use of oxytocine was the same in both groups (Table 3).

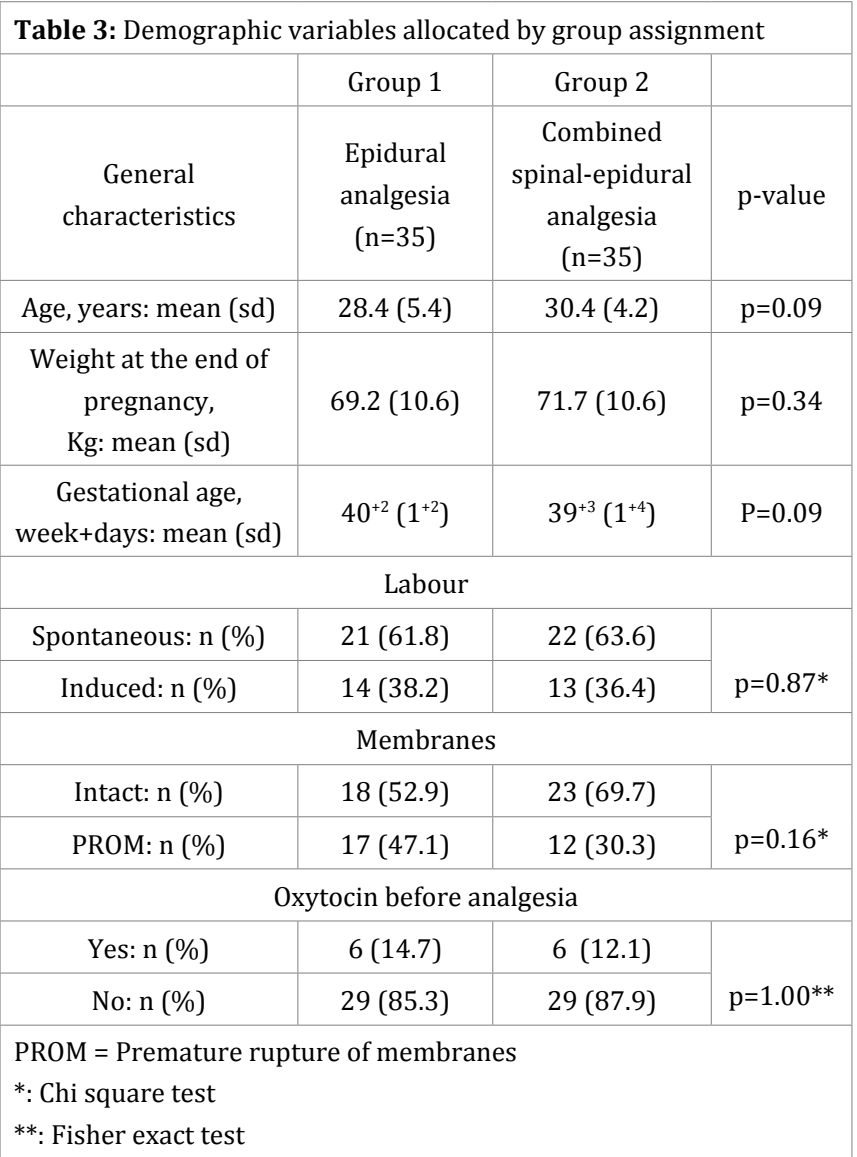

\section{Hemodynamic impact}

Both groups exhibited a minimal and non statistically significant decrease from the baseline SAP $(127,9+14,6 \mathrm{mmHg}$ in EA and 124,6+28,3 mmHg in CSEA) at 15 minutes (Figure 2),

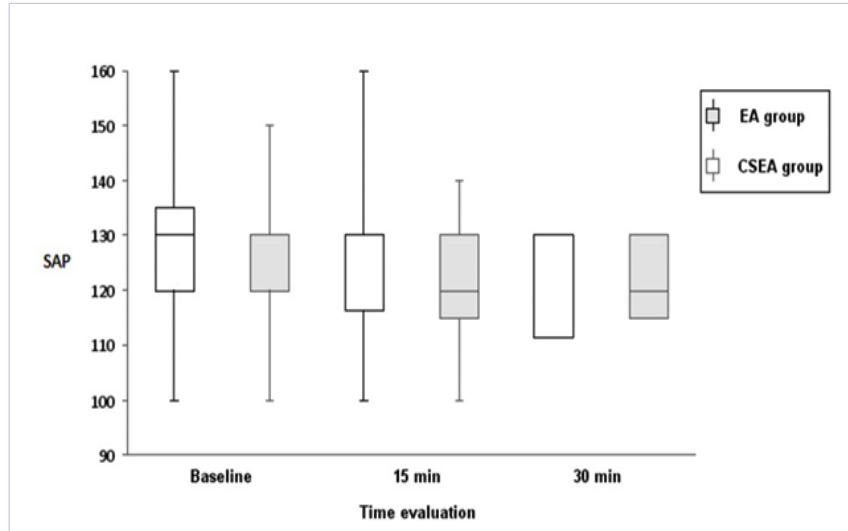

Figure 2: Comparison of hemodynamic impact EA vs CSEA group.

with no differences between the two groups $(125,9+15,54 \mathrm{mmHg}$ in EA and $122+14,62 \mathrm{mmHg}$ in CSEA); no further reduction was noted even at 30 minutes and until delivery. No episodes of hypotension were reported.

\section{Quality of analgesia}

As described in Fig. 3, NRS at the enrolment was equal to 9 or 10 for most of the parturients $(88 \%$ and $85 \%$ in EA and CSEA group, respectively). In both groups we observed a significant reduction of NRS mean values from 15 minutes after the start of the analgesia, with further reduction at 30 minutes $(\mathrm{p}<0,05)$. However, NRS was lower in CSEA group already at 15 minutes $(3,2+2,4$ vs 5,1+2,2 in EA group) and also at 30 minutes $(2+2$ vs $3,2+1,6$ in EA group) ( $p=0,003)$.

\section{Effects on labor and delivery}

We found no significant differences between the two groups with regards to the total time for dilatation, time for dilatation after analgesia, expulsive period, use and dose of oxytocin and delivery types. The total dose of sufentanil was significantly higher in the EA group (Table 4).

\section{Side effects}

We did not observe nausea, vomiting, maternal hyperthermia, hypothension or urinary retention in any group. In EA group, 3 patients (9\%) complained of pruritus (severe

Table 4: Effects of epidural and combined spinal epidural analgesia on labor duration and delivery

\begin{tabular}{|c|c|c|c|}
\hline Use of oxytocin after analgesia & Epidural analgesia & Combined spinal-epidural analgesia & $19 / 16$ \\
\hline Yes/No: $\mathrm{n}$ & $18 / 17$ & $2.7(4.7)$ \\
\hline Dose of oxytocin, u: mean (\%) & $2.8(3.0)$ & $284.5(122.2)$ & $16.90^{*}$ \\
\hline Duration of dilatation,min: mean & $302.9(158.4)$ & $180.2(103.6)$ & $13.3(20.7)$ \\
\hline Dilatation with analgesia,min: mean (\%) & $209.7(118.0)$ & $47.7(28.6)$ \\
\hline Latency period, min: mean (sd) & $13.1(18.6)$ & $\mathrm{p}=0.44^{* *}$ & $\mathrm{p}=0.48^{+}$ \\
\hline Expulsive period, min: mean (sd) & $51.2(34.6)$ & $\mathrm{p}=0.86^{* *}$ & \\
\hline Type of delivery & & \\
\hline
\end{tabular}




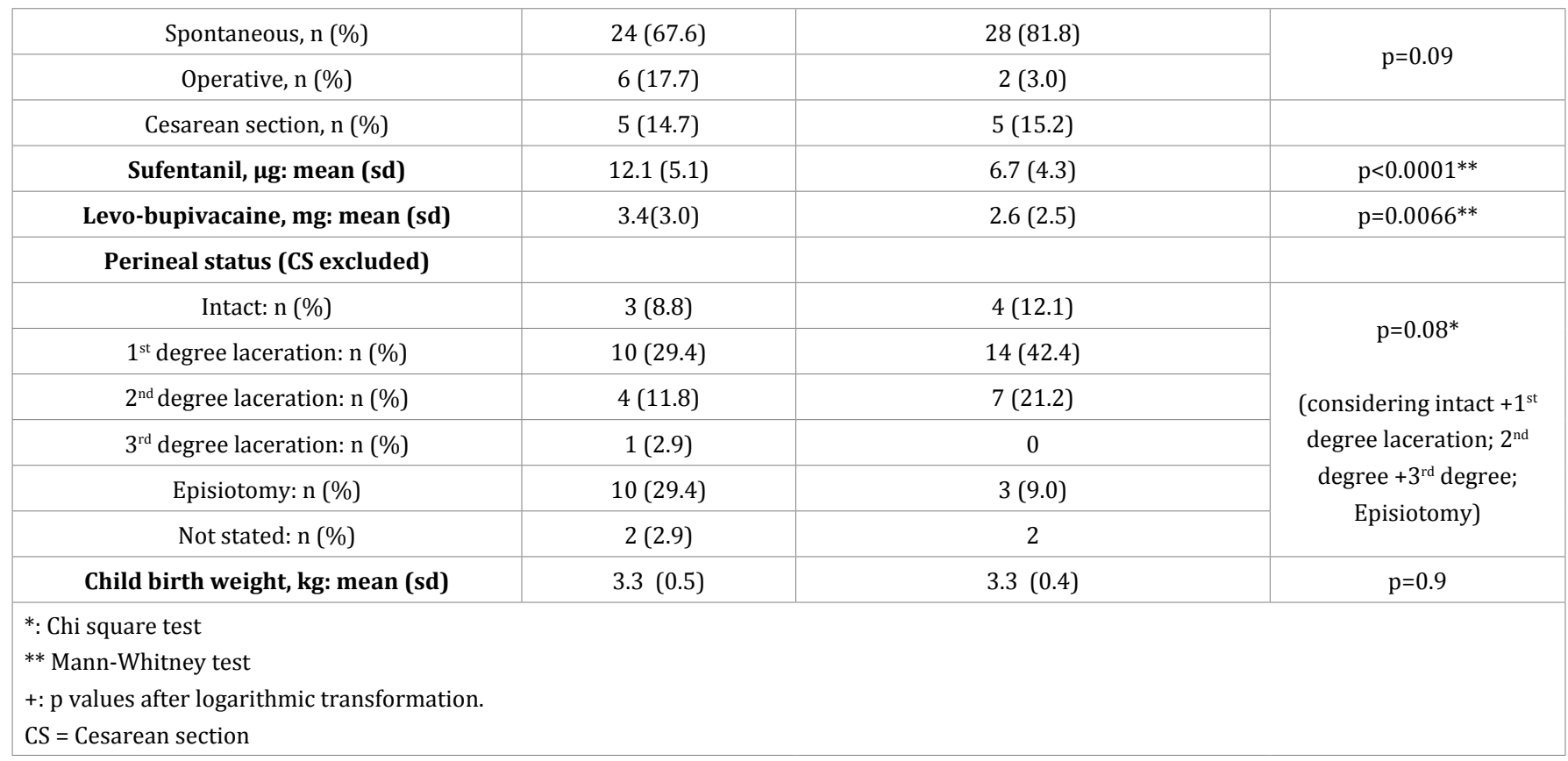

in 1 case) compared to 15 (45\%) in CSEA group (severe in 5) $(\mathrm{p}<0,005)$. In EA group uterine hypertonia was recorded in 5 cases $(14 \%) ; 2(5,7 \%)$ cases of uterine hyperkinesia (presence of more than 6 uterine contractions in 10 minutes) with fetal bradycardia in 2 cases. In CSEA group, only one episode of hypercinesia $(2,8 \%)$ and one case of hypertonia (persistence of uterine contraction for more than 3 minutes) associated to fetal bradycardia were recorded.

Clinically significant FHR abnormalities were similar between the groups. In the first 20 minutes after injection, a non-reassuring FHR was observed in $6(17 \%)$ cases in EA group (decelerations in 5 cases and reduction of variability in one case that rapidly improved without medical intervention in 20 to 40 minutes interval). In CSEA group, a non-reassuring FHR (recurrent variable decelerations) was recorded in $4(11 \%)$ cases; only in one case abnormalities persisted until birth (delivery within one hour interval after analgesia) without any negative impact on the newborn. At childbirth, an umbilical cord turn was evident.

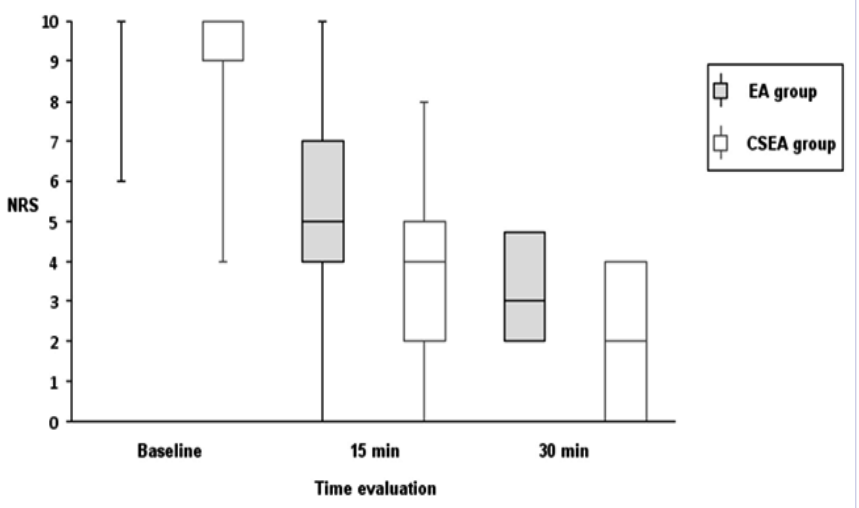

Figure 3: Comparison of quality of analgesia EA vs CSEA group.
In the EA group, only 1 (2,8\%) case of fetal bradycardia was noted that resolved spontaneously within few minutes. In CSEA group, $3(8,8 \%)$ cases of pathological FHR (bradycardia: FHR $<110$ bpm for more than 2 minutes) were noted, one of which (persistent) resulted in a cesarean section delivery, the other two resolved within 2 minutes.

No differences in neonatal outcome were observed between the two groups with a mean Apgar score at birth and after 5 minutes of 8/9 in the EA group and 9/9 in CSEA group.

\section{Discussion}

While sufentanil is gaining popularity worldwide as opioid agent for analgesia during the labor, it has been argued that some side effects of opioids might be more intense after intrathecal administration [19]. In the specific case of sufentanil, its possible local anesthetic effect and rapid analgesic action after intrathecal administration have been proposed as plausible causes of hypotension during the labor [10]. Our study found no difference in the hemodynamic effects of neuraxial analgesia with intrathecal vs epidural sufentanil in the early stage of the labor. Interestingly, at the time of this writing, studies comparing the effects of the same opioid after intrathecal and epidural administration of equianalgesic doses were scarce [20] and no studies comparing the side-effects of the epidural and intrathecal ED50 of sufentanil at the early stage of the labor could be retrieved [11]. It is noteworthy that our study showed no hemodynamic differences between the two groups but faster reduction of NRS values in CSEA group at 15 min when exposed to two equianalgesic regimens; this may suggest an additional advantage for CSEA, as it would provide faster and better analgesia at the first stage of labour with no added hemodynamic repercussions. In line with our results, a recent Cochrane Review that compared CSEA and EA involving 3274 women found no difference in maternal hypotension whereas a more rapid onset of analgesia in the CSEA

Citation:Alba Ripoll Gallardo, et.al. (2017) Hemodynamic Impact of Intrathecal versus Epidural Analgesia with Sufentanil in 
group [21]. Also, we found no significant differences between the two groups regarding urinary retention and rates of operative or instrumental deliveries. On the contrary, the above mentioned Cochrane Review found higher rates of these two side effects when pain relief was provided with epidural analgesia; this could be due to the fact that our study included a smaller number of women. Remarkably, pruritus has been even defined as the "hallmark of intrathecal opioid", reaching an incidence $>80 \%$ [22]. Accordingly, in our study pruritus was also more common in CSEA group. While it has been argued that the administration of intrathecal opioids during the first stage of labor may determine fetal heart rate abnormalities $[23,24]$, other authors have concluded that more evidence is needed to designate spinal opioids as cause of a higher incidence of fetal heart rate alterations [25]. Our findings may support the assertion that significant FHR abnormalities are rare when low doses of intrathecal sufentanil are administered [5].

An acute decrease in maternal plasma epinephrine levels (due to analgesia) may cause a temporary imbalance of uterinetocolytic/tocodynamic forces usually associated with uterine hypertonia [26]. This seemed to be related to the rapidity of analgesic effect and, as confirmed by Van der Velde, it is probably a dose-related effect [5]. It is noteworthy that, even if a greater and faster reduction of NRS values was achieved in the CSEA group, the total number of hypercinesia episodes was lower. It may be due to the fact that the total dose of sufentanil injected was lower in CSEA group. Our study found no difference in the hemodynamic impact of the median effective dose of sufentanil at the early stage of the labor when intrathecal and epidural route were compared. This fact together with the faster onset of CSEA and similar relevant side effects of EA and CSEA, may favor the utilization of CSEA at the early stage of the labor.

\section{Limitations}

This randomized controlled trial was restricted to uncomplicated nulliparous patients in an attempt to obtain the best uniformity of population in study and to reduce the number of confounding factors that may influence hemodynamic values. Our results should therefore be interpreted carefully in patients with higher ASA scores. This was a single blinded study because only patients were blinded to the anesthetic technique (EA vs CSEA). Noteworthy, the evaluation of the hemodynamic impact, throughout the measurement of the systolic blood pressure and the collection of the NRS might be considered as an objective value and patient-blinded data respectively.

The hemodynamic parameters were recorded 15 minutes after the first bolus and successively every 30 minutes untill delivery; using 15 and 30 minutes may have missed the detection of minimum hemodynamic changes on the arterial blood pressure and heart rate, which may have been clinically significant. This study is powered to evaluate the impact on maternal hemodynamics and so, the confirmation of a statistically significant difference in pain control between the two regimens will require further studies.

\section{Conclusions}

\section{Ethical approval}

Ethical approval for this study (Ethical Committee $n$ 170/09) was provided by the Ethical Committee of the Azienda Ospedaliera Universitaria "Maggiore della Carità" Novara, Italy on December 18, 2009 - Chairperson: Dr. Gianfranco Zulian. This clinical trial study was registered as EudraCT:2009-017665-31.

\section{Availability of data and material}

The datasets during and/or analysed during the current study available from the corresponding author on reasonable request.

\section{Competing interests}

There are no situations in which this manuscript may be perceived as conflict of interest or as a copyright constraint.

\section{Authors' contributions}

-G .M. hasseen the original study data,reviewed the analysis of thedata, contributed to the article writing and approved the final manuscript.

-A.R.G. reviewed the analysis of the data, contributed to the article writing and approved the final manuscript.

-C.R. has seen the original study data, reviewed the analysis of the data, and approved the final manuscript.

-V.V has seen the original study data, reviewed the analysis of the data, approved the final manuscript, and is the author responsible for archiving the study files.

-S.F. has seen the original study data, reviewed the analysis of the data, approved the final manuscript, and is the author responsible for archiving the study files.

-D.F. carried out the statistical analysis.

-F.D.C. has seen the original study data, reviewed the analysis of the data, and approved the final manuscript.

\section{Acknowledgements}

The authors are expressing their thanks and gratitude for the support, assistance and collaboration of the medical and nursing staff of the Obstetric and Gynecology department of the Maggiore della Carità during this study. We thank also Dr. Tudor Codreanu for the English revision of the text.

\section{Trial registration:}

EudraCT:2009-017665-31. Registered December 2009

\section{References}

1. Anim-Somuah M, Smyth RM, Jones L. Epidural versus nonepidural or no analgesia in labour. Cochrane Database Syst Rev . 2011;(12):CD000331.

2. Kuczkowski KM. Labor pain and its management with the combined spinal- epidural analgesia:what does an obstetrician need to know. Arch Gynecol Obstet. 2007;275(3):183-185.

3. Nelson KE, Rauch T,Terebuh V, D'Angelo R. A comparison of intrathecal fentanyl and sufentanil for labor analgesia. Anesthesiology. 


\section{2;96(5):1070-1073.}

4. Lilker S, Rofaeel A, Balki M, Carvalho JC. Comparison of fentanyl and sufentanil as adjuncts to bupivacaine for labor epidural analgesia. J Clin Anesth. 2009;21(2):108-112.

5. Van de Velde M, Teunkens A, Hanssens M,Vandermeersch E Verhaeghe J. Intrathecal sufentanil and fetal heart rate abnormalities: a double-blind, double placebo-controlled trial comparing two forms of combined spinal epidural analgesia with epidural analgesia in labor. Anesth Analg. 2004;98(4):1153-1159.

6. Capogna G,Camorcia M, Columb MO. Minimum analgesic doses of fentanyl and sufentanil for epidural analgesia in the first stage of labor Anesth Analg. 2003;96(4):1178-1182.

7. Gaiser RR, Cheek TG, Gutsche BB. Comparison of three different doses of intrathecal fentanyl and sufentanil for labor analgesia. J Clin Anesth. 1998;10(6):488-493.

8. Salem IC, Fukushima FB, Nakamura G,Ferrari F,Navarro LC, Castiglia YM, et al. Side effects of subarachnoid and epidural sufentanil associated with a local anesthetic in patients undergoing labor analgesia. Rev Bras Anestesiol. 2007;57(2):125-135.

9. Riley ET, Ratner EF, Cohen SE. Intrathecal sufentanil for labor analgesia: do sensory changes predict better analgesia and greater hypotension. Anesth Analg. 1997; 84(2):346-351.

10. Riley ET, Walker D, Hamilton CL,Cohen SE. Intrathecal sufentanil for labor analgesia does not cause a sympathectomy. Anesthesiology. 1997;87(4):874-878

11. Camann WR, Denney RA, Holby ED, Datta S. A comparison of intrathecal, epidural, and intravenous sufentanil for labor analgesia. Anesthesiology . 1992;77(5):884-887.

12. Herman NL, Calicott R,Van Decar TK, Conlin G, Tilton J. Determination of the dose-response relationship for intrathecal sufentanil in laboring patients. Anesth Analg. 1997;84(6):1256-1261.

13. Bujedo BM. Current evidence for spinal opioid selection in postoperative pain. Korean J Pain. 2014;27(3):200-209.

14. Birnbach DJ, Davidson EM. Pain relief during labor. In: Kurjak A Chervenak FA.editors.Textbook of Perinatal Medicine. Second Edition. Vol1. London. 1998:1879

15. Royal College of Obstetricians and Gynecologists (RCOG) - The use of Electronic fetal Monitoring. Evidence-based Clinical Guidelines Number 8. RCOG Clinical Effectiveness support unit LRP

16. Ministero della Salute. "Taglio cesareo: una scelta appropriata e consapevole." 2011.

17. Bromage PR. Epidural Analgesia. PSW. 1978

18. Littell RC, Henry PR, Ammerman CB. Statistical analysis of repeated measures data using SAS procedures. J Anim Sci. 1998;76(4):12161231

19. Chaney MA. Side effects of intrathecal and epidural opioids. Can J Anaesth. 1995;42(10):891-903.

20. Ipe S, Korula S, Varma S, George GM, Abraham SP, Koshy LR, et al. A comparative study of intrathecal and epidural buprenorphine using combined spinal-epidural technique for caesarean section. Indian ] Anaesth. 2010;54(3):205-209.

21. Simmons SW,Taghizadeh N, Dennis AT, Hughes D, Cyna AM. Combined spinal- epidural versus epidural analgesia in labour. Cochrane Database Syst Rev . 2012;10:CD003401.
22. DeBalli P, Breen TW. Intrathecal opioids for combined spinal-epidural analgesia during labour. CNS Drugs. 2003;17(12):889-904.

23. Van de Velde M, Vercauteren M, Vandermeersch E. Fetal heart rate abnormalities after regional analgesia for labor pain: the effect of intrathecal opioids. Reg Anesth Pain Med.2001;26(3):257-262.

24. Mardirosoff C,Dumont L, Boulvain M, Tramer MR. Fetal bradycardia due to intrathecal opioids for labour analgesia: a systematic review. BJOG . 2002;109(3):274-281.

25. Norris MC. Intrathecal opioids and fetal bradycardia: is there a link? Internatl J Obstet Anesth. 2000;9:264-269.

26. Hughes SC. Clinical Applications of Intraspinal Analgesia in Obstetrics. Levinson's Anesthesia for Obstetrics. Fourth edition. Philadelphia. 2002;162. 